\title{
Video case: Dense Infiltration by Rhabitiform Larvae of Strongyloides stercoralis during Childhood
}

\author{
Feleke Eriso \\ Parasitologist,Dilla University, Dilla, Ethiopia \\ feleke.eriso@yahoo.com
}

The densely concentrated number of rhabditiform larvae of Strongyloides stercoralis was isolated from a fresh stools sample of a 13 years old male child. The stools sample was centrifuged at the speed of $1000 \mathrm{rpm}$ (revolutions of the rotor per minute) for 2 minutes. The supernatant was discarded and then about 3 drops of the supernatant was added to the sediment at the bottom of each centrifuge tube that were shaked well. Now, the shaked suspensions of the sediment were collected in a vial.
Next, a drop of the shaked suspension was placed on a clean slide with the help of a dropper and covered with a cover slip. After that, the preparation was focused on under the low power objective lens of the compound light microscope and the motile rhabditiform larvae you have seen were recorded with a digital camera from the field of vision of the microscope. There were no serious clinical manifestations with the child from whom this heavy load of rhabditiform larvae was obtained. The child was treated and cured successfully with a single dose of $6 \mathrm{mg}$ ivermectin tablet on an empty stomach. 